\title{
Secreted miR-34a in astrocytic shedding vesicles enhanced the vulnerability of dopaminergic neurons to neurotoxins by targeting Bcl-2
}

\author{
Susu Mao ${ }^{1,2}$, Qi Sun ${ }^{1,2,3}$, Hui Xiao ${ }^{1,2}$, Chenyu Zhang ${ }^{1,2 \bowtie}$, Liang $\mathrm{Li}^{1,2 \bowtie}$ \\ ${ }^{1}$ State Key Laboratory of Pharmaceutical Biotechnology, Nanjing Advanced Institute for Life Sciences (NAILS), \\ Nanjing University School of Life Sciences, Nanjing 210093, China \\ 2 Jiangsu Engineering Research Center for microRNA Biology and Biotechnology, Nanjing 210093, China \\ ${ }^{3}$ Center of Kidney Disease, 2nd Affiliated Hospital, Nanjing Medical University, Nanjing 210003, China \\ $\square$ Correspondence: cyzhang@nju.edu.cn (C. Zhang), lijing@sibs.ac.cn (L. Li) \\ Received February 10, 2015 Accepted April 16, 2015
}

\begin{abstract}
MicroRNAs (miRNAs) are a class of noncoding RNAs that regulates target gene expression at posttranscriptional level, leading to further biological functions. We have demonstrated that microvesicles (MVs) can deliver miRNAs into target cells as a novel way of intercellular communication. It is reported that in central nervous system, glial cells release MVs, which modulate neuronal function in normal condition. To elucidate the potential role of glial MVs in disease, we evaluated the effects of secreted astrocytic MVs on stress condition. Our results demonstrated that after Lipopolysaccharide (LPS) stimulation, astrocytes released shedding vesicles (SVs) that enhanced vulnerability of dopaminergic neurons to neurotoxin. Further investigation showed that increased astrocytic miR-34a in SVs was involved in this progress via targeting anti-apoptotic protein $\mathrm{Bcl}-2$ in dopaminergic neurons. We also found that inhibition of astrocytic miR-34a after LPS stimulation can postpone dopaminergic neuron loss under neurotoxin stress. These data revealed a novel mechanism underlying astrocyte-neuron interaction in disease.
\end{abstract}

Susu Mao, Qi Sun contributed equally to this work.

Electronic supplementary material The online version of this article (doi:10.1007/s13238-015-0168-y) contains supplementary material, which is available to authorized users.
KEYWORDS astrocyte, shedding vesicles, miR-34a, dopaminergic neurons, Bcl-2

\section{INTRODUCTION}

Parkinson's disease (PD) is characterized by the progressive degeneration of dopamine (DA) neurons in the substantia nigra (SN). Circumstantial evidence has shown that the neuron-glia interaction plays an important role in PD pathogenesis (Furman and Norris, 2014; Lee et al., 2013; Oeckl et al., 2012). It has been suggested that reactive astrocytes may amplify the devastating effects of PD by accelerating the dopaminergic (DA) neuron loss (Saijo et al., 2009).

Microvesicles (MVs) were originally described as cellular debris with limited biological function (Morel et al., 2004). However, increasing amounts of evidence suggest that MV shedding is a constitutive mode of intercellular communication (Al-Nedawi et al., 2009; Gupta and Pulliam, 2014; Hu et al., 2012; Keller et al., 2006; Shantsila et al., 2010). MVs can be divided into two groups-shedding vesicles (SVs) and exosomes-with different discharging processes (Bianco et al., 2009). The release of shedding vesicles is a result of direct budding from the plasma membrane, while exosomes are produced by exocytosis of multivesicular bodies (Zomer et al., 2010). Bianco F et al. showed that in the central nervous system, glial cells can release both type of MVs (Bianco et al., 2009). Further investigation produced evidence that MVs released from microglia stimulate synaptic activity via enhanced sphingolipid metabolism, indicating that microglia MVs may have significant biological functions under normal 
conditions (Antonucci et al., 2012). Our previous work showed that exosomes can deliver microRNAs (miRNAs) into endothelial cells and then modulate the migration of the cell by repressing the target protein expression (Zhang et al., 2010). In recent years, accumulating evidence also showed that secreted miRNAs in MVs have significant biological functions including effects on cell proliferation, development, differentiation as well as cell death and cancer progression (Hu et al., 2012; Janowska-Wieczorek et al., 2005; Jung and Suh, 2014; Liu et al., 2013; Vlassov et al., 2012; Zhou et al., 2014; Zhu and Fan, 2011). However, there are few studies regarding the function of secreted miRNAs in neuron-glia interaction of the central nervous system either in physiological or disease conditions. Whether secreted miRNAs in astrocytic MVs are involved in PD pathogenesis is still unknown; therefore it is important to investigate the potential role of MVs in astrocyteneuron interactions in PD.

In the present study, we used both in vitro and in vivo PD models to evaluate the effects of secreted astrocytic MVs under different conditions on DA neurons survival. We demonstrated that astrocytes under conditions of LPS stress released SVs, which enhanced the vulnerability of DA neurons to neurotoxin. We also found evidence that miR-34a was increased in astrocytic SVs after LPS stimulation and that miR-34a then entered DA cells and repressed the anti-apoptotic protein Bcl-2 (Hockenbery et al., 1993; Wang et al., 2009; Yang et al., 1997; Zhou et al., 2014), thus compromising cellular resistance to neurotoxins. In addition, we showed that blocking the astrocytic miR34a can rescue the anti-apoptotic function of DA neurons in vitro and alleviate DA neuron loss as well as abnormal behavior induced by apomorphine under 6-OHDA stress in vivo. These data suggest a new mechanism by which astrocytes influence neuronal survival under disease conditions.

\section{RESULTS}

Biochemical and morphological characterization of distinct types of microvesicles released from astrocytic cells

We collected the shedding vesicles (SVs) and exosomes from the culture medium by differential ultracentrifugation. EM imaging showed the different sizes of the distinct types of microvesicles. SVs consisted of cup-shaped vesicles in the range of $100-200 \mathrm{~nm}$, while exosomes contained relatively small vesicles of approximately 30-80 nm (Fig. 1A and 1B).

We also analyzed the different types of vesicles by Western blotting of some SVs and exosome markers. Typical SV markers, such as $\beta 1$ integrin and ribophorin, were enriched in our 10,000 $\times g$ pellet, while exosome markers, such as CD63 and heat shock protein HSP70, were found mainly in the $110,000 \times g$ pellet. Furthermore, we also found Ago2 protein expression in both types of the releasing microvesicles (Fig. 1C). Ago2 is reported to be associated with functional miRNAs, indicating that the released microvesicles might have carried miRNAs with Ago2 proteins.

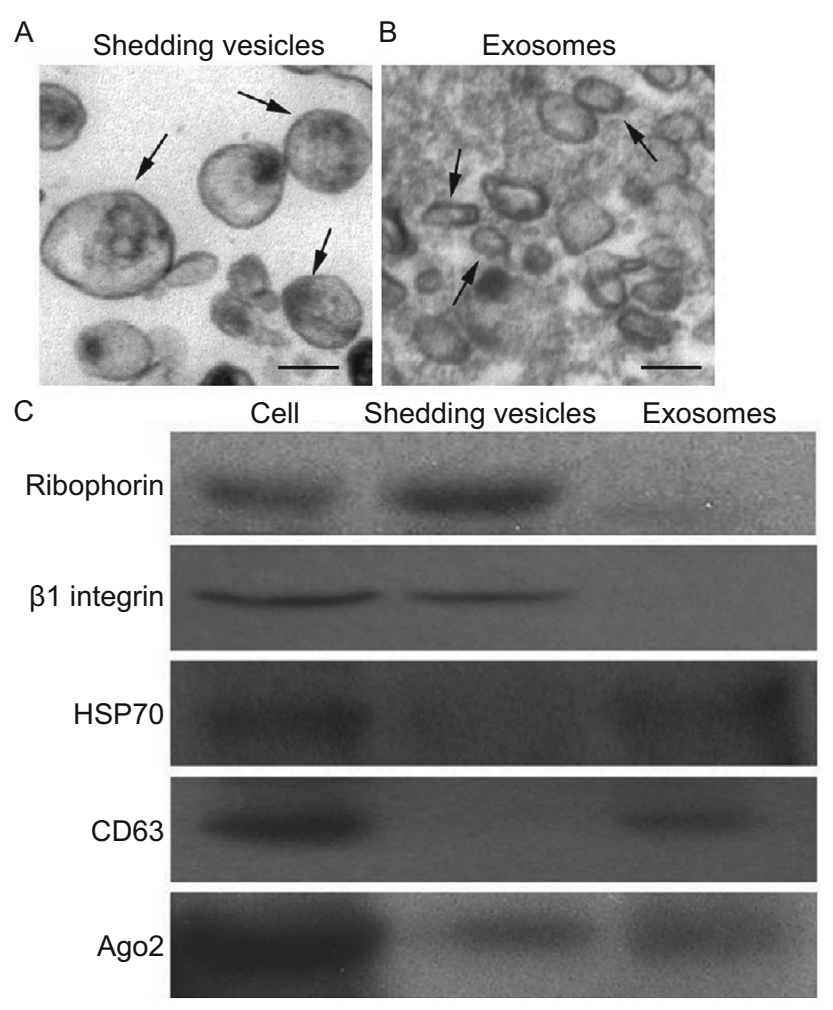

Figure 1. Morphology and characterization of purified microvesicles of U-87 MG cells. (A) EM imaging of purified shedding vesicles secreted by U-87 MG cells (arrows, scale bar $=100 \mathrm{~nm}$ ); (B) EM imaging of purified exosomes secreted by U-87 MG cells (arrows, scale bar = $100 \mathrm{~nm}$ ); (C) Western blot of the molecular markers of shedding vesicles (Ribophorin and $\beta 1$ integrin) and exosomes (CD63 and HSP70); both types of microvesicles contained the miRNA-associated protein Ago2.

\section{Astrocyte-derived shedding vesicles under stress conditions enhanced the vulnerability of DA neurons to neurotoxins}

Next, we investigated the impact of astrocyte-derived microvesicles under stress conditions on cell survival. We found that neither SVs nor exosomes derived from the LPSstimulated U87-MG astroglial cell line had any effects on $\mathrm{SH}$ SY5Y cell viability under normal conditions (Fig. S1). However, further investigation showed that pretreatment of SVs derived from LPS-stimulated U87-MG cells increased the vulnerability of the SH-SY5Y cells to threshold concentrations of neurotoxins, such as $0.2 \mathrm{mmol} / \mathrm{L} \mathrm{MPP+}$ or $10 \mu \mathrm{mol} / \mathrm{L}$ 6-OHDA (Figs. 2A, 2B and S1).

\section{MiRNA profiling of SVs derived from LPS-stimulated U-87 MG cells}

We collected the SVs from LPS (LPS SVs) and PBS (control SV) treated U-87 MG cells, respectively. In the present study, 
A
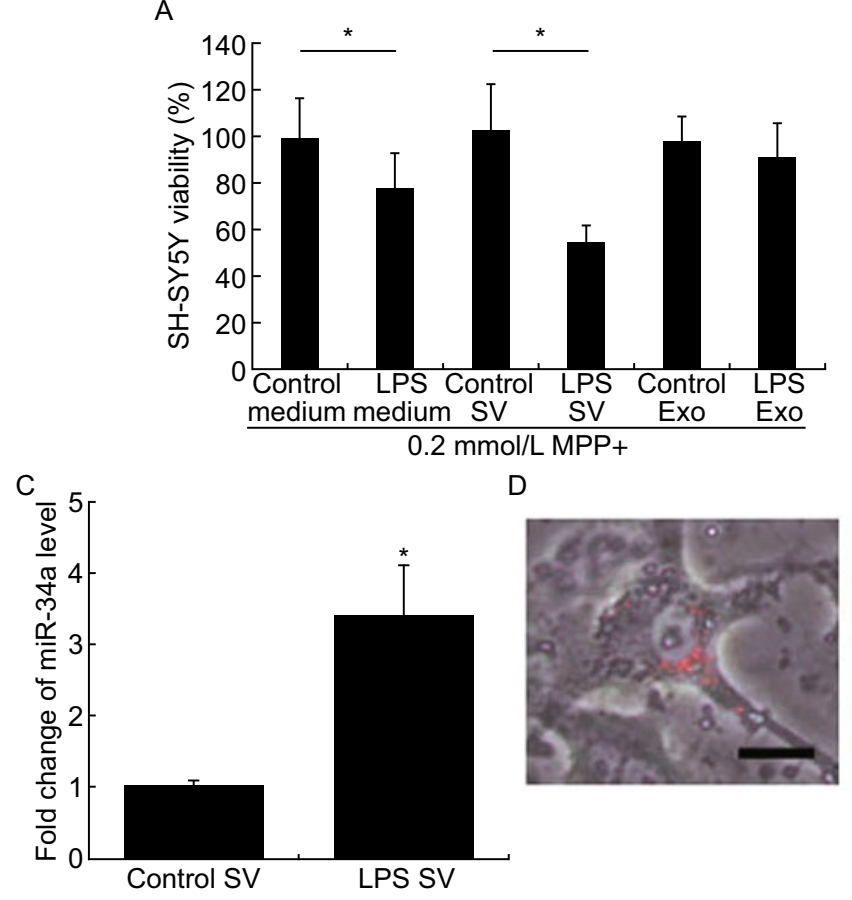

B

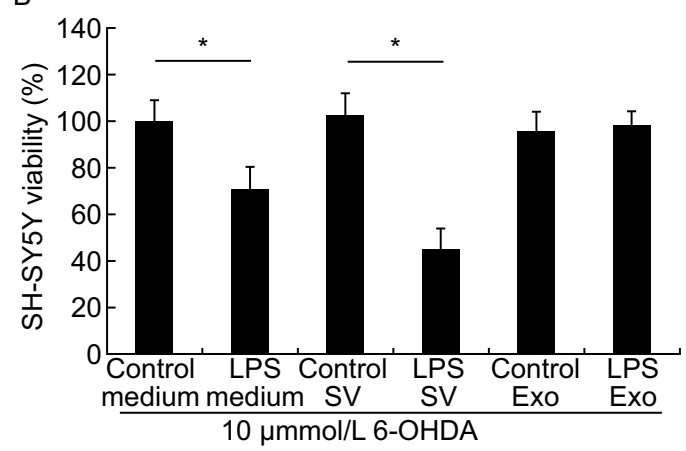

$\mathrm{E}$

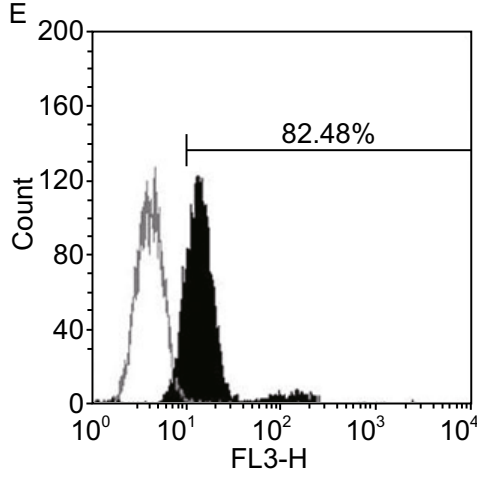

Figure 2. SVs derived from LPS-stimulated U-87 MG cells contain increased miR-34a and reduce SH-SY5Y cell viability to neurotoxins. (A) Cell viability assessed by CCK-8 showed that pretreatment with LPS SVs increased the vulnerability of SH-SY5Y cells to $0.2 \mathrm{mmol} / \mathrm{L} \mathrm{MPP+}$ treatment, ${ }^{*} P<0.05$; (B) Cell viability assessed by CCK-8 showed that pretreatment with LPS SVs increased the vulnerability of SH-SY5Y cells to $10 \mu \mathrm{mol} / \mathrm{L} 6-\mathrm{OHDA}$ treatment, ${ }^{*} P<0.05$. Control SV: SVs derived from PBSstimulated U-87 MG cells; LPS SVs: SVs derived from LPS-stimulated U-87 MG cells; Control Exo: Exosomes derived from control U-87 MG cells; LPS Exo: Exosomes derived from LPS-stimulated U-87 MG cells; (C) Relative miRNA levels between control SV and LPS SVs, * $P<0.05$; (D) Fluorescent images showed that labeled SVs derived from U-87 MG cells can enter SH-SY5Y cells after coincubation; red dots are labeled shedding vesicles, Scale bar $=10 \mu \mathrm{m}$; (E) FACS analysis showed that more than $80 \%$ of SH-SY5Y cells carry red fluorescence after co-incubation with labeled SVs; the left histogram represents the control group; the right histogram represents SH-SY5Y cells co-cultured with the fluorescence-labeled SVs.

we first analyzed the miRNA levels in LPS SV and control SV using miRNA array. We focused on those miRNAs with a fold-change (LPS SV/control SV) $>2$ or $<0.5$, respectively. In each group, 10 miRNAs were listed by their signal intensity in a descending manner (Table S1). Among these altered miRNAs profiles, miR-34a was of particular interest, as it was upregulated 2.86-fold in LPS SV, and more so, as one of its predicted genes, $\mathrm{Bcl}-2$, has been demonstrated to regulate cell apoptosis (Hockenbery et al., 1993; Yang et al., 1997). We further assessed the expression level of miR-34a in LPS SV by qPCR. Consistent with the result of miRNA array, we found that miR-34a was significantly increased, with a fold change of $3.4 \pm 0.7$ (Fig. 2C). Because it has been reported that miR-34a targets $\mathrm{Bcl}-2$ protein, which regulates cell apoptosis, we proposed that up-regulation of miR-34a in LPS SVs may contribute to the increased vulnerability of SHSY5Y cells to neurotoxins via repressing Bcl-2 expression.
Secreted miR-34a in LPS SVs enhanced the vulnerability of SH-SY5Y cells to neurotoxins by repressing $\mathrm{Bcl}-2$ protein expression

We co-cultured the fluorescence-labeled SVs of U-87 MG cells with SH-SY5Y cells and found labeled SVs in cultured SH-SY5Y cells by fluorescence microscopy (Fig. 2D). FACS analysis also showed that the $82.48 \%$ of cultured SH-SY5Y cells were positive for fluorescence-labeled SVs (Fig. 2E). These results indicated the possibility that SVs may be able to deliver miRNAs into target cells by co-culture.

As it has been reported that miR-34a may target Bcl-2 protein, we first performed the luciferase assay and Western blot in SH-SY5Y cells which showed that miR-34a can indeed target $\mathrm{Bcl}-2 \mathrm{mRNA}$ directly and repress $\mathrm{Bcl}-2$ protein levels in SH-SY5Y cells (Fig. S2). Next, we further investigated the role of secreted miR-34a in neuronal loss under 

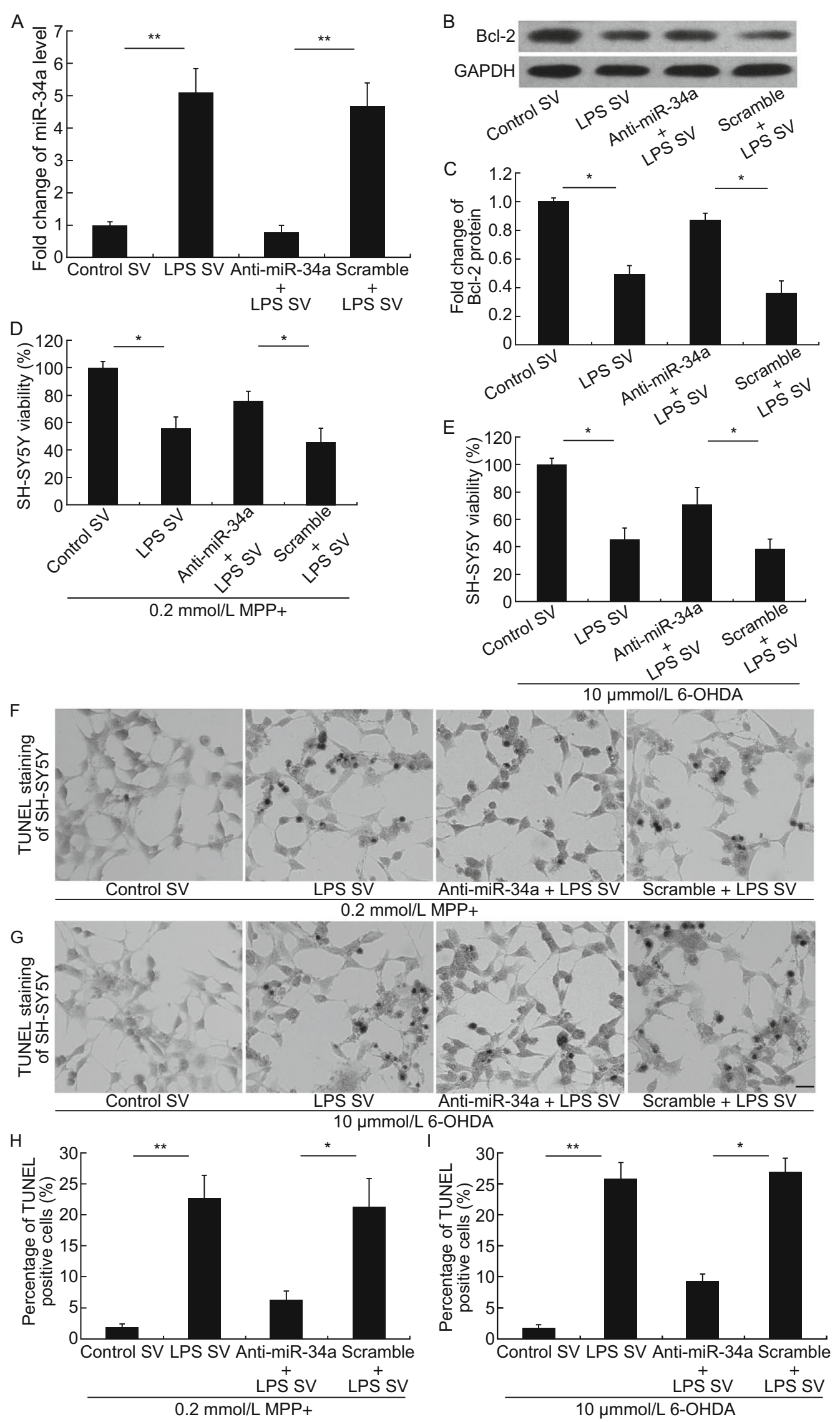
Figure 3. Co-incubation with LPS SVs increases miR-34a level while down-regulates $\mathrm{Bcl}-2$ level that further compromises neurotoxin resistance of SH-SY5Y cells. (A) QPCR analysis of miR-34a levels in SH-SY5Y cells after co-incubation with different groups of SVs, ${ }^{* *} P<0.01$; (B and C) Western-blot and quantification of Bcl-2 protein levels in SH-SY5Y cells after coincubation with different groups of SVs, ${ }^{*} P<0.05$; (D and E) Viability of SH-SY5Y cells pre-treated with different groups of SVs following $0.2 \mathrm{mmol} / \mathrm{L} \mathrm{MPP+}$ or $10 \mu \mathrm{mol} / \mathrm{L}$ 6-OHDA stress, ${ }^{*} P<0.05$; $(\mathrm{F}$ and $\mathrm{G})$ TUNEL staining of $\mathrm{SH}-\mathrm{SY} 5 \mathrm{Y}$ cells pre-treated with different groups of SVs after $0.2 \mathrm{mmol} / \mathrm{L} \mathrm{MPP}+$ or $10 \mu \mathrm{mol} / \mathrm{L}$ 6-OHDA stress, scale bar $=50 \mu \mathrm{m} ;(\mathrm{H}$ and $\mathrm{I})$ Percentage of TUNEL positive cells among SH-SY5Y cells pre-treated with different types of SVs after $0.2 \mathrm{mmol} / \mathrm{L} \mathrm{MPP+}$ or $10 \mu \mathrm{mol} / \mathrm{L}$ 6-OHDA stress, ${ }^{*} P<0.05,{ }^{* \star} P<0.01$; Control SV: SVs derived from control U-87 MG cells; LPS SV: SVs derived from LPSstimulated U-87 MG cells; anti miR-34a + LPS SV: SVs derived from LPS-stimulated U-87 MG cells transfected with miR-34a inhibitor; Scramble + LPS SV: SVs derived from LPS-stimulated U-87 MG cells transfected with scramble RNA.

neurotoxin treatment. We found that the level of mature miR34a was significantly increased in SH-SY5Y cells after the treatment with LPS SVs (Fig. 3A). As a result, the protein level of $\mathrm{Bcl}-2$ was repressed in these cells (Fig. $3 \mathrm{~B}$ and $3 \mathrm{C}$ ). However, pretreatment with anti-miR-34a in U-87 MG cells blocked the increase of miR-34a and negated the repression of Bcl-2 caused by LPS SVs (Fig. 3A-C). Further investigation showed that cellular viability was reduced and apoptotic ratio was increased in LPS SV-treated SH-SY5Y cells after $0.2 \mathrm{mmol} / \mathrm{L} \mathrm{MPP}+$ exposure. On the other hand, pretreating U-87 MG cells with anti-miR-34a significantly lessened the effect of LPS SVs on SH-SY5Y cell viability and apoptotic ratio (Fig. $3 \mathrm{D}, 3 \mathrm{~F}$ and $3 \mathrm{H}$ ). Similar results were achieved when 6-OHDA was used (Fig. 3E, 3G and 3I).

In addition, we transfected miR-34a into U-87 MG cells and then collected the SVs (miR-34a SVs) without LPS stimulation. In these SVs, miR-34a level was increased by approximately $22.1 \pm 2.7$ fold (Fig. S3). Pretreatment with miR-34a SVs increased the levels of mature miR-34a in SHSY5Y cells, leading to a decrease in Bcl-2 protein levels (Fig. 4A-C). Moreover, we also observed that miR-34a SVs reduced the cellular viability and increased the percentage of apoptotic SH-SY5Y cells after $0.2 \mathrm{mmol} / \mathrm{L} \mathrm{MPP}+$ or $10 \mu \mathrm{mol} / \mathrm{L}$ 6-OHDA treatment (Fig. 4D-I).

Together, these data provide strong evidence that secreted miR-34a in LPS SVs plays a major role in regulating apoptosis of SH-SY5Y cells exposed to neurotoxins by repressing Bcl-2 protein expression.

\section{SVs derived from LPS-stimulated astrocytes increased primary DA neuron loss after neurotoxin exposure}

Here, we further used primary culture to verify the results demonstrated in the cell lines. We collected LPS SVs from primary astrocytes and found that the miR-34a levels were also increased (Fig. S4). Pretreatment with primary astrocytic LPS SVs caused significant TH-positive cell loss after low concentration MPP+ $(0.1 \mu \mathrm{mol} / \mathrm{L})$ or $6-\mathrm{OHDA}(2 \mu \mathrm{mol} / \mathrm{L})$ treatment, without significant effect on the total neuron number showed by DAPI (Fig. 5A-D). This effect was strongly attenuated after blocking the up-regulation of miR34a in astrocytic LPS SVs by anti-miR-34a pretreatment in astrocytes. To further verify that miR-34a is necessary and sufficient to induce apoptosis, we also used antagomiR oligonucleotides directed against miR-34a (antagomiR-34a) to block the level of miR-34a in primary neurons. Similar results were obtained (Fig. 5A-D). These results indicated that in an ex vivo situation, stressed astrocytes released SVs that increased TH neuron vulnerability, in which an increased level of secreted miR-34a may be involved.

\section{Secreted miR-34a in LPS SVs enhanced the DA neuron vulnerability to 6-OHDA in vivo}

To further understand the role of LPS SVs in vivo, we evaluated the effects of different groups of SVs in the 6-OHDA rat model. We injected 6-OHDA unilaterally into the striatum while simultaneously injecting SVs into the SN in the same side (Fig. 6A). We found that the number of DA neurons was significantly decreased in the LPS SVs-treated rats after 1 week, while blocking the up-regulation of miR-34a in LPS SVs can reduce the DA neuron loss at the same time point. However, when we examined DA neurons 3 weeks after the injection, we found that all groups of rats showed severe DA neuron loss with no significant differences (Figs. 6B and 2C). We further assessed apomorphine-induced contralateral rotation of these rat groups at different time points ( 1 week, 2 weeks and 3 weeks respectively) after the surgery. Among 15 rats for each group that exhibit abnormal apomorphine-induced rotation at the third week $(>6 / \mathrm{min})$, we found that the probability of disease onset of the anti-miR-34a + LPS SV group is $3 / 15$ on the first week and $5 / 15$ on the second week while the numbers in scramble + LPS SV group are $8 / 15$ and 11/15 respectively (Fig. 6D). These results showed that blocking the up-regulation of miR-34a in LPS SVs can significantly delay the disease onset but not able to reverse the disease progression after the injection.

Taken together, our data suggest that LPS-stimulated astrocyte SVs can enhance DA neuron vulnerability, which accelerates the progression of DA neuron loss, and that miR-34a plays a crucial role in this process.

\section{DISCUSSION}

Neuron-glia interaction is thought to play an important role in the pathogenesis of many neurodegenerative diseases (Malavolta et al., 2013; Saijo et al., 2009). In the present study, we revealed a novel mechanism by which astrocytes can influence neuronal survival under PD conditions. Our results 
demonstrated that reactive astrocytes release SVs (not exosomes), which increase the percentage of apoptotic SH-SY5Y cells after treatment with low concentrations of neurotoxins. Thus, these SVs may increase DA neuron vulnerability. Furthermore, we demonstrated that there are differences in miRNA expression patterns between SVs produced by reactive and resting astrocytes and that miR-34a was significantly up-regulated. Our previous work demonstrated that MVs can deliver miRNAs into target cells and shut down target gene expression (Zhang et al., 2010). In addition, several studies revealed that MVs mediated cell interaction exists in central nervous system (Antonucci et al., 2012; Hu et al., 2012). Moreover, there is evidence that miR-34a targets the antiapoptotic protein, $\mathrm{Bcl}-2$, which can further induce tubular cell apoptosis (Liu et al., 2011; Sun et al., 2012; Wang et al., 2009; Zhou et al., 2014). Here we also performed luciferase assay which ensure that miR-34a can directly bind and regulate $\mathrm{Bcl}-$ 2 in SH-SY5Y cells. These data together indicate the possibility that astrocytic miR-34a may be transferred into neurons via SVs, where it then represses Bcl-2 protein levels and decreases the anti-apoptotic function of the targeted neurons. Notably, several other miRNAs listed in Table S1 also have important functions in modulating cell apoptosis. Among upregulated miRNAs, miR-18a was validated to regulate cell apoptosis by targeting Bcl-2 directly in mice and humans (Scherr et al., 2014), and miR-296-3p was found to increase apoptosis levels of an islet cell line $\alpha \mathrm{TC} 1-6$ cells (Barbagallo et al., 2013). On the other hand, in those down-regulated miRNAs, inhibition of miR-708 promoted the apoptosis of SW480 cells (Lei et al., 2014) and T24 cells (Song et al., 2013). These data suggest that in addition to miR-34a, those altered miRNAs summarized in Table S1 may also affect cell apoptosis. Nevertheless, our data demonstrated that the level and variation of miR-34a in astrocytic SVs is one of the highest among these miRNAs upon LPS stimulation, which indicates that miR-34a plays a major role in modulating cell apoptosis.

Furthermore, we provided direct evidence that fluorescence-marked astrocytic SVs can enter target cells after $24 \mathrm{~h}$ of incubation, increase miR-34a levels and decrease $\mathrm{Bcl}-2$ protein levels in SH-SY5Y cells. Blocking the increase in the miR-34a levels in astrocytic SVs by a miR-34a inhibitor alleviated the DA cell loss caused by LPS-stressed astrocytic SVs. This evidence indicates that increased miR-34a levels may contribute to the disruptive effect of reactive-astrocytic SVs on DA cell survival. Since our miRNAs array data indicated that the level of several other miRNAs also changed in astrocytic SVs after LPS stimulation, we further testify the effect of miR-34a by over-expression experiment. When we increased the miR-34a levels in astrocytic SVs without LPS stimulation by transfecting astrocytes with premiR-34a, we found that those SVs still enhanced the vulnerability of DA neurons to low concentrations of neurotoxins, leading to an increased percentage of apoptotic cells. These results strongly indicated that the upregulation of miR$34 a$ in astrocytic SVs is sufficient to increase neuronal vulnerability under disease conditions.
Figure 4. Overexpression of miR-34a in SVs is sufficient to increase the vulnerability of SH-SY5Y cells to neurotoxins. (A) QPCR analysis of miR-34a levels in $\mathrm{SH}$ SY5Y cells after co-incubation with miR-34a SV compared with cells co-incubated with control SV, ${ }^{*} P<0.05$; (B and C) Western-blot and quantification of Bcl-2 protein levels in SH-SY5Y cells after co-incubation with miR-34a SV compared with cells co-incubated with control SV, ${ }^{*} P<0.05$; (D and E) Viability of SH-SY5Y cells pre-treated with different groups of SVs after $0.2 \mathrm{mmol} / \mathrm{L} \mathrm{MPP}+$ or 10 $\mu \mathrm{mol} / \mathrm{L}$ 6-OHDA stress, ${ }^{*} P<0.05$; (F and H) TUNEL staining of SH-SY5Y cells pre-treated with different groups of SVs after $0.2 \mathrm{mmol} / \mathrm{L} \mathrm{MPP+}$ or $10 \mu \mathrm{mol} / \mathrm{L} 6-\mathrm{OHDA}$ stress, scale bar $=50 \mu \mathrm{m}$; (G and I) Percentage of TUNEL positive cells of SH-SY5Y cells pre-treated with different types of SVs after $0.2 \mathrm{mmol} / \mathrm{L} \mathrm{MPP}+$ or $10 \mu \mathrm{mol} / \mathrm{L} 6-\mathrm{OHDA}$ stress, ${ }^{*} P<0.05,{ }^{* *} P<0.01$. Control SV: SVs derived from control U-87 MG cells; miR-34a SV: SVs derived from U-87 MG cells that overexpressed miR-34a.

In primary culture, we found that LPS-stimulated rat astrocytes also generated SVs with higher levels of miR-34a. In addition, SVs derived from LPS-stressed primary astrocytes enhanced primary DA neuron vulnerability to low concentrations of both MPP+ and 6-OHDA. This disruptive effect was alleviated when we blocked the increase of miR$34 a$ using a miR-34a inhibitor. All of these results were consistent with the data obtained from the cell lines, which suggests that a similar mechanism exists for both primary and cell line culture systems.

Next, we investigated the role of secreted miR-34a in astrocytic SVs in regulating neuron survival in vivo. Using the 6-OHDA model, we demonstrated that SVs derived from LPS-stimulated primary astrocytes significantly accelerated DA neuron loss following 6-OHDA treatment. Most of the rats lost more than $30 \%$ of their DA neurons in the first week and showed apomorphine-induced rotation. When we blocked the increase of miR-34a in astrocytic SVs by transfecting miR-34a inhibitor into primary astrocytes, we found that the acceleration of DA neuron loss was reduced and that apomorphine-induced rotation was delayed compared with scramble group. These data indicate that the increased levels of secreted miR-34a in LPS-stimulated-astrocytic SVs play a crucial role in the acceleration of DA neuron loss. Recently, it is reported that miR-34a is shuttled in exosomes which induces cell apoptosis in fibrotic kidney (Zhou et al., 2014). This is similar with our findings in nervous system except that in our model we demonstrated that SVs contain the increase of miR-34a and display robust effect rather than exosomes in regulating cell apoptosis. When we examined those PD rats three weeks after 6-OHDA injection, we found all groups showed severe DA neuron loss. Combined with behavioral tests, it is indicated that blocking the function of secreted miR-34a in LPS-stimulated astrocytic SVs can postpone but not reverse the progress of disease. 

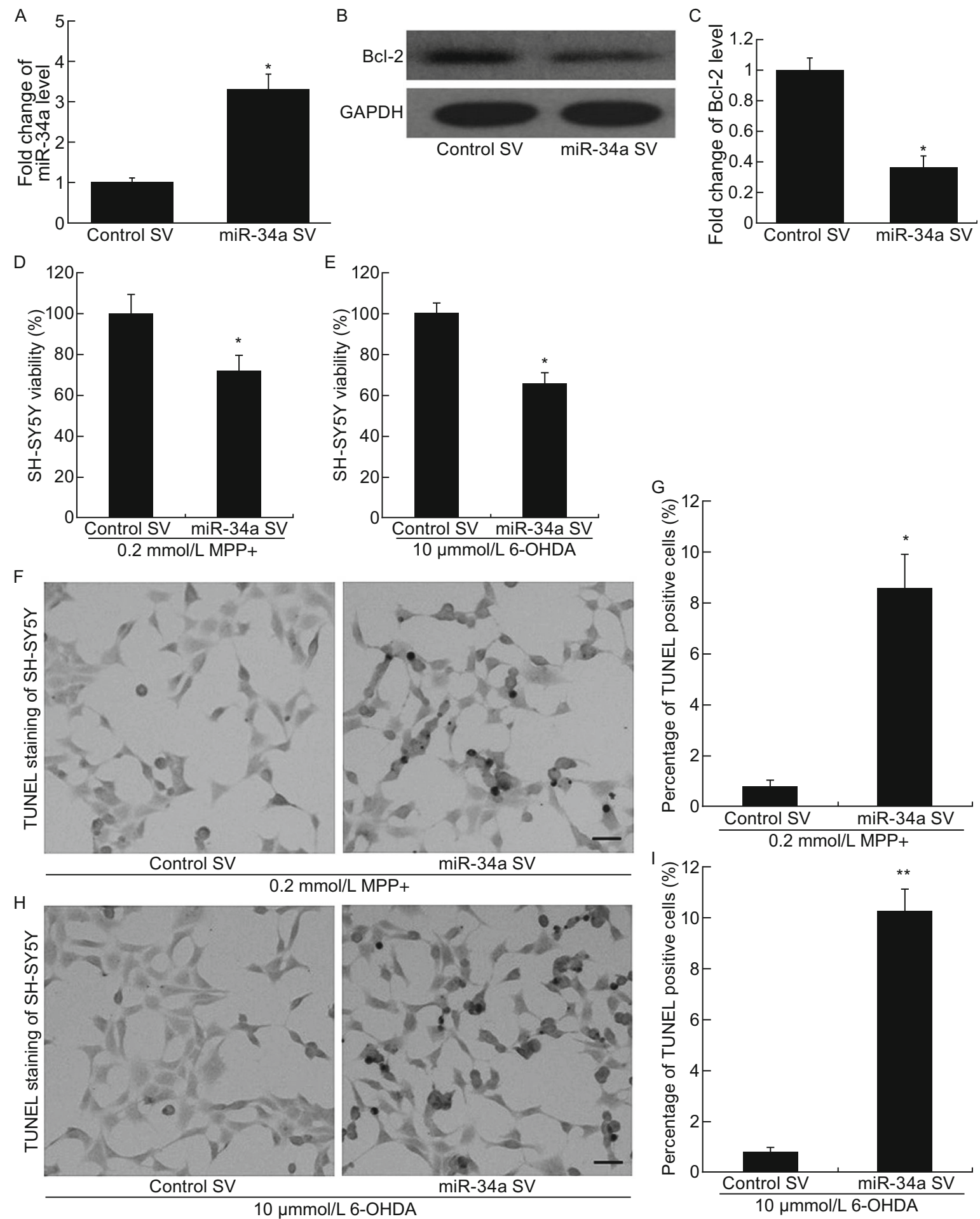
A

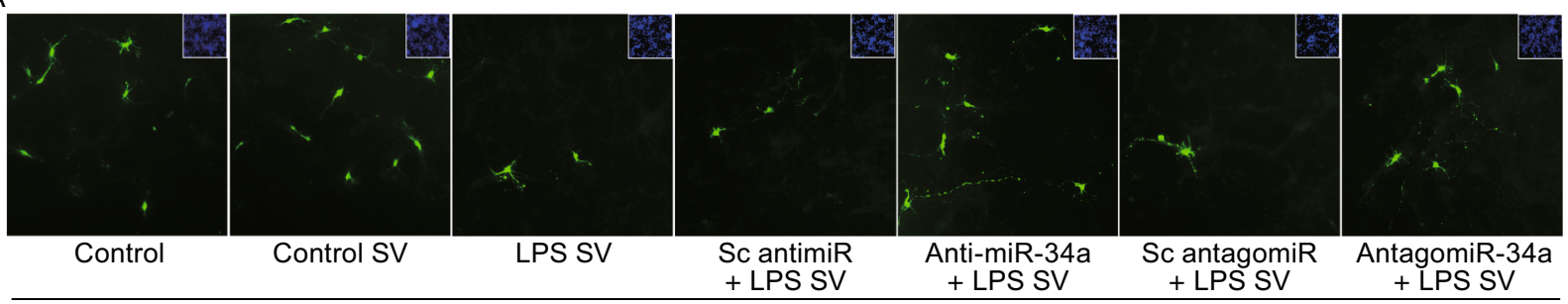

B

$0.1 \mu \mathrm{mol} / \mathrm{L} \mathrm{MPP}+$
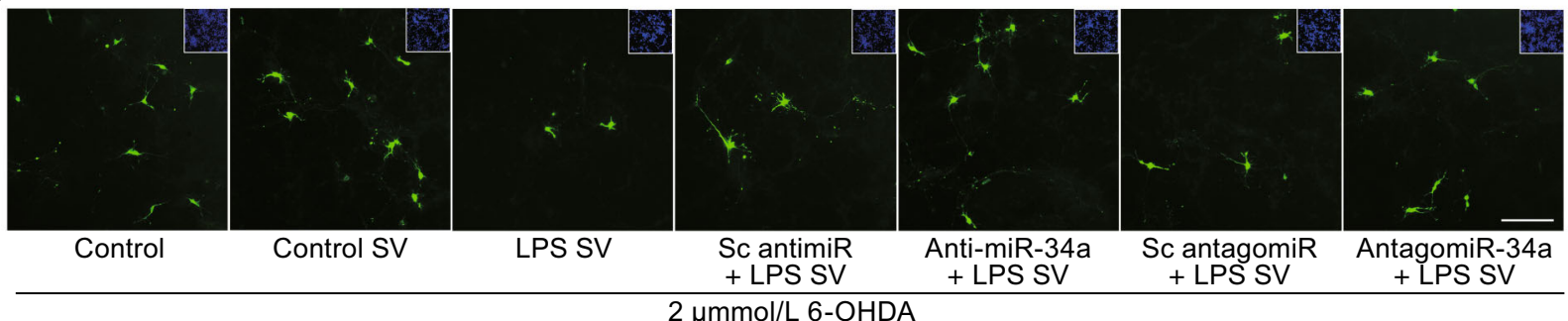

C

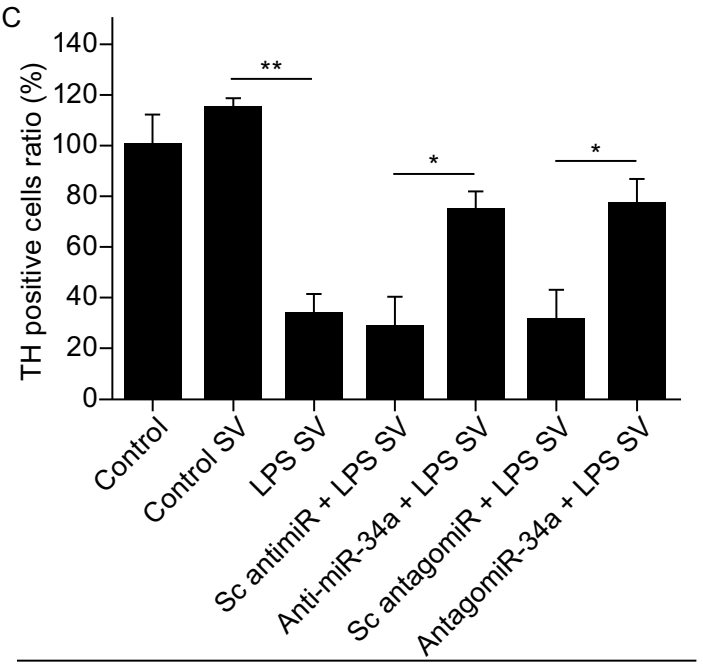

D

$0.1 \mu \mathrm{mol} / \mathrm{L} \mathrm{MPP}+$

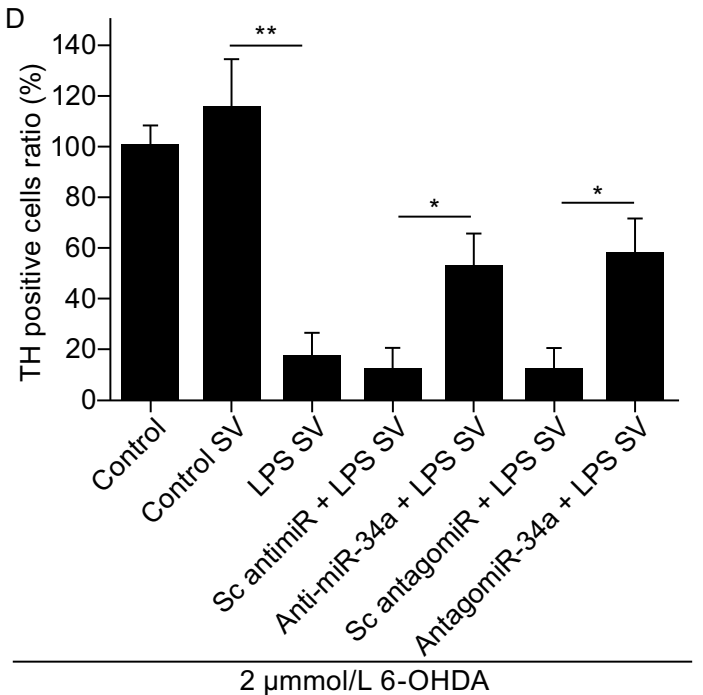

Figure 5. SVs derived from LPS-stimulated primary astrocytes enhance DA neurons loss after treatment with neurotoxins. (A and C) TH staining and quantification of the percentage of TH-positive cells among primary neurons pre-treated with different groups of SVs after $0.1 \mu \mathrm{mol} / \mathrm{L} \mathrm{MPP}+$ stress, ${ }^{*} P<0.05$, ${ }^{* *} P<0.01$. Inset: DAPI staining of the cell nuclei in the field of view. (B and D) $\mathrm{TH}$ staining and quantification of the percentage of TH-positive cells among primary neurons pre-treated with different groups of SVs after $2 \mu \mathrm{mol} / \mathrm{L} 6-\mathrm{OHDA}$ stress, ${ }^{*} P<0.05,{ }^{* \star} P<0.01$, scale bar $=200 \mu \mathrm{m}$. Inset: DAPI staining of the cell nuclei in the field of view. Control: a control group of neuron culture without SVs incubation; Control SV: SVs derived from primary astrocytes; LPS SV: SVs derived from LPS-stimulated primary astrocytes; anti-miR-34a + LPS SV: SVs derived from LPS-stimulated primary astrocytes transfected with miR-34a inhibitor; Sc antimiR + LPS SV: SVs derived from LPS-stimulated primary astrocytes transfected with scramble RNA; Sc antagomiR + LPS SV: SVs derived from LPS-stimulated primary astrocytes, and the primary neurons were pretreated with scramble antagomiR; antagomiR-34a + LPS SV: SVs derived from LPS-stimulated primary astrocytes, and the primary neurons were pretreated with antagomiR-34a.

In many but not all cases of Parkinson's disease, an increase in the number of reactive astrocytes has been observed (Forno et al., 1992), which suggest that astrocytes play vital roles in PD. In the 6-OHDA animal model of PD, Stromberg found an increased amount of reactive astrocytes in the striatum (Stromberg et al., 1986). Activation of astrocytes is a prevalent response to neuronal damage in neurodegenerative diseases, with potential neurotoxic and neuroprotective consequences. Several findings suggest a neuroprotective role for astrocytes in PD as well as animal models of PD. Saura et al. found infusion of IL-1 $\beta$ led to an increased activation of astrocytes in the the substantia nigra and the dopaminergic neurons were protected 
A

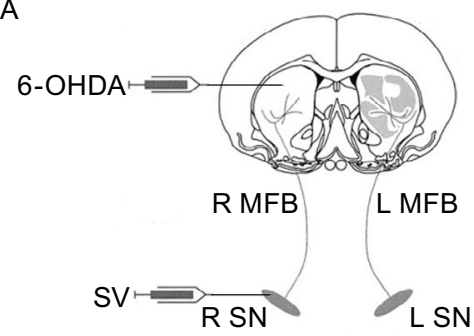

B

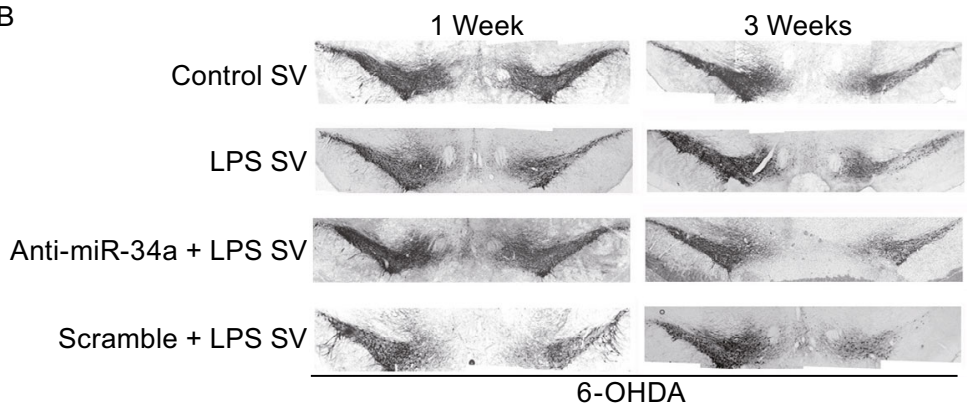

D

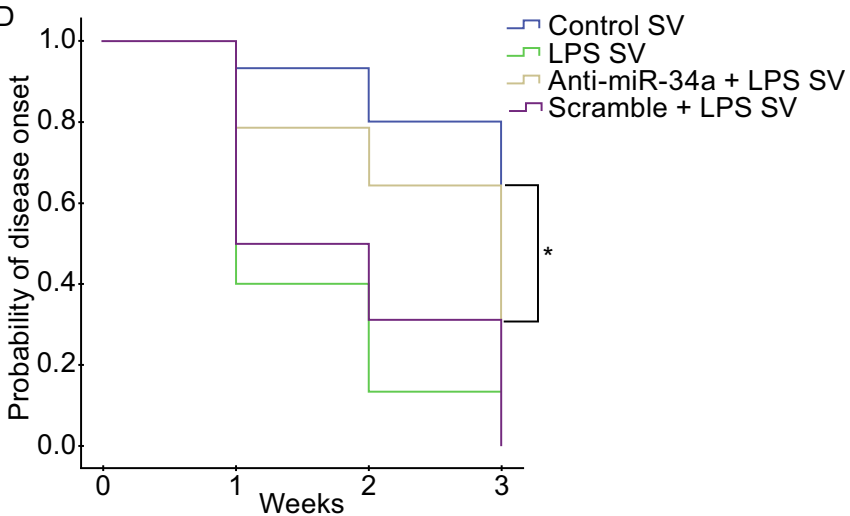

Figure 6. SVs derived from LPS-stimulated primary astrocytes accelerates DA neurons loss as well as disease onset in 6-OHDA rat model. (A) Schematic diagram of the experiment process; (B) TH-immunoreactive cell bodies in SN of different groups of rats; (C) Quantification of DA neurons loss among different groups of rats, ${ }^{*} P<0.05$ ( $n=10$ in each group); (D) The Kaplan-Meier curves demonstrate the probability of disease onset, ${ }^{*} P<0.05$. ( $n=15$ in each group).

against 6-OHDA (Saura et al., 2003). It also has been shown that astrocytes can protect neurons from $\mathrm{NO}$ toxicity by a glutathione dependent mechanism (Chen et al., 2001). Additionally, astrocytes express monoamine oxidase-B and catecholO-methyl-transferaseare, thus reducing the oxidative stress by metabolizing dopamine (Hirsch et al., 1999). On the other hand, $\mathrm{Li}$ et al. found that suitable activation of astrocytes increased their protection effect on dopaminergic neurons stimulated by LPS, while excessive activation attenuated it, which suggested that astrocytes played a double-faced effect on dopaminergic neurons ( $\mathrm{Li}$ et al., 2009). Also, astrocytes can release chemokines and cytokines that are deleterious to neurons in various models of $P D$, such as reactive oxygen species, nitrite, S100B and so on (Niranjan et al., 2010). The present study suggested that astrocytes had a destructive effect through SVmiR-34a-Bcl2 pathway under LPS stress condition. These results suggest that in stress condition, activated astrocytes may act as a double-edged sword in regulating DA neurons survival. Such functional balance of reactive astrocytes needs to be further explored. Altogether, our results suggested that under stress conditions, such as LPS stimulation, increased levels of miR-34a in astrocytic SVs can be transported into DA neurons, where they enhance the vulnerability of the neurons to neurotoxins by targeting the anti-apoptotic protein Bcl-2. The increase of astrocytic miR-34a in disease conditions may be a potential target for alleviation of PD progress.

\section{MATERIALS AND METHODS}

Animals and cell culture

All of the animal care and experimental procedures were performed in accordance with the Laboratory Animal Care Guidelines approved by the Model Animal Research Center of Nanjing University. U-87 MG and SH-SY5Y cell lines were maintained in Dulbecco's Modified Eagle Medium: Nutrient Mixture F-12 (DMEM/F-12, Life Technologies, Grand Island, NY, USA) with 10\% Fetal Bovine Serum (FBS, Life Technologies). Primary cultures of rat brain astrocytes were prepared as follows: briefly, newborn (P0) rat cerebral cortices were separated in Hank's Balanced Salt Solution (HBSS, Life Technologies) and digested into cell suspensions. Digestion was terminated by the addition of $10 \%$ FBS. Cells were seeded in $75 \mathrm{~cm}^{2}$ flask and 
maintained in DMEM/F-12 medium containing 10\% FBS. After 14 days of culture, the astrocytes were separated from the microglia and oligodendrocytes by shaking for $12 \mathrm{~h}$ in an orbital shaker at $240 \mathrm{rpm}$. Cell samples were checked regularly for the expression of glial fibrillary acid protein, a marker for astrocytes. Only those cultures containing $>95 \%$ astrocytes were used. Primary cultures of rat DA neurons were prepared as follows: briefly, embryonic (E14.5) rat ventral mesencephalon (VM) was removed from the brains in HBSS and digested into cell suspensions. Digestion was terminated by the addition of $10 \%$ FBS. Cells were seeded in 24-well plates that were coated with $0.05 \%$ poly-D-lysine (Sigma-Aldrich, St Louis, MO) and maintained in neurobasal medium (Life Technologies) containing $2 \%$ B27 (Life Technologies) and $0.2 \mathrm{mmol} / \mathrm{L}$ L-glutamine (Life Technologies) for 4 more days in vitro before use. U-87 MG cells and primary astrocytes were treated with different concentrations of LPS (Sigma-Aldrich) for $6 \mathrm{~h}$ and washed three times with PBS. The conditioned medium was collected after another $48 \mathrm{~h}$ culture. SH-SY5Y cells and primary DA neurons were treated with different groups of astrocytic SVs in an astrocyte-to-neuron ratio of 9:1 for $24 \mathrm{~h}$ before exposure to neurotoxin. SH-SY5Y cells were exposed to different concentrations of 1-Methyl-4-phenylpyridinium iodide (MPP+, Sigma-Aldrich) or 6-Hydroxydopamine hydrobromide (6-OHDA, Sigma-Aldrich) for $6 \mathrm{~h}$ and cultured for another $24 \mathrm{~h}$ before subsequent assays. Primary DA neurons were exposed to different concentrations of MPP+ or 6-OHDA for $1 \mathrm{~h}$ and cultured for another $24 \mathrm{~h}$ before subsequent assays.

\section{MVs isolation and electronic microscopy}

MVs were isolated from culture medium by differential centrifugation as in previous publications (Bianco et al., 2009). After removing cells and other debris by centrifugation at $300 \times g$ and $1200 \times g$, the supernatant was centrifuged at $10,000 \times g$ for $1 \mathrm{~h}$ to collect the shedding vesicles (SVs) and at $110,000 \times g$ for $2 \mathrm{~h}$ to collect the exosomes; all steps were performed at $4^{\circ} \mathrm{C}$. MVs were collected from the pellet and resuspended in FBS-free medium. In preparation for Electron Microscope (EM) imaging, MVs were precipitated, fixed in a $0.1 \mathrm{~mol} / \mathrm{L}$ phosphate buffer containing $2.5 \%$ glutaraldehyde (Sigma-Aldrich), and cut into $50 \mu \mathrm{m}$-thick sections using a vibratome. The sections were postfixed with $1 \% \mathrm{OsO}_{4}$, dehydrated and embedded in Durcupan (ACM; Fluka, Buchs, Switzerland) on a microscope slide and covered with a coverslip. The sections were cut again using a Reichert ultramicrotome into 70-nm-thick sections. The ultrathin sections were then stained with uranyl acetate and lead citrate and evaluated with an electron microscope.

RNA isolation, microRNA array and quantitative real-time PCR of mature miRNAs

The total RNA of the MVs derived from $10^{8}$ cells was extracted using TRIzol Reagent (Life Technologies). Samples of total RNA were extracted for miRNAs Affymetrix GeneChip miRNA 3.0 array analysis (Life Technologies). Quantitative real-time PCR (qPCR) was performed using TaqMan microRNA probes (Applied Biosystems, Foster City, CA, USA) according to the manufacturer's instructions. Briefly, $1 \mu \mathrm{g}$ of the total RNA was reverse-transcribed to produce cDNA using Avian Myeloblastosis Virus reverse transcriptase
(Takara, Dalian, China) and stem-loop RT primers (Applied Biosystems). Real-time PCR was performed using a TaqMan PCR kit and an Applied Biosystems 7300 Sequence Detection System (Applied Biosystems). All of the reactions, including the no-template controls, were run in triplicate. After the reactions, the CT values were determined using fixed-threshold settings.

\section{Fluorescence labeling of SVs}

U-87 MG cells were treated with SynaptoGreen (Sigma-Aldrich) for $6 \mathrm{~h}$ and washed five times with PBS; after the cells were cultured for another $48 \mathrm{~h}$, the medium was collected and the SVs were isolated. The collected SVs were added into the SH-SY5Y cells in an astrocyte-to-neuron ratio of 9:1 for $24 \mathrm{~h}$. Subsequently, the SH-SY5Y cells were collected and analyzed by fluoromicroscopy or Fluorescence Activated Cell Sorter (FACS).

\section{Cell transfection with ncRNA, anti-miR-34a or pre-miR-34a}

U-87 MG cells, primary astrocytes and primary neurons were transfected using Lipofectamine 2000 (Life Technologies) according to the manufacturer's instructions. For overexpression of miR-34a, $50 \mathrm{nmol} / \mathrm{L}$ of pre-miR-34a or scrambled negative control pre-miRNA (pre-ncRNA) was used. For knockdown of miR-34a, $20 \mathrm{nmol} / \mathrm{L}$ of anti-miR-34a, $50 \mathrm{nmol} / \mathrm{L}$ of antagomiR oligonucleotides directed against miR-34a or scrambled negative control anti-miRNA (antincRNA) was used. Cell media were collected $48 \mathrm{~h}$ after transfection.

\section{Luciferase assay}

The $3^{\prime} \mathrm{UTR}$ of $\mathrm{Bcl}-2$ containing the predicted target sequence was cloned and inserted into pMIR-REPORT ${ }^{\mathrm{TM}}$-Luciferase vector (Ambion, Austin, TX, USA). Forward primer: 5'-CATGCCTGCCCCAAACAAATA-3'; reverse primer: 5'-AGGGCATTTTTCCCATCGCT-3'. Mutated vector was generated in Invitrogen by replacing the predicted target region with its reverse sequence (from ACUGCC to TGACGG). $\mathrm{SH}-\mathrm{SY} 5 \mathrm{Y}$ cells were seeded in 24 well plates for $12 \mathrm{~h}$. Afterwards, 0.2 $\mu \mathrm{g}$ of firefly luciferase reporter plasmid, $0.2 \mu \mathrm{g}$ of $\beta$-galactosidase ( $\beta$ gal) expression vector (Ambion), and $50 \mathrm{nmol} / \mathrm{L}$ of miR-34a mimics or scrambled control were transfected into cells. Cells were harvested for luciferase assay (Promega, Madison, WI, USA) $24 \mathrm{~h}$ later, and luciferase activities were normalized to $\beta$-gal activities.

\section{CCK-8 Assay}

Cytotoxicity was also assayed using CCK-8 (Dojindo, Kumamoto, Japan) assay. Briefly, SH-SY5Y cells were cultured in a 96-well plate with different treatments. The cells were then treated with $10 \mu \mathrm{L}$ CCK- 8 solution per well and incubated for $3 \mathrm{~h}$ at $37^{\circ} \mathrm{C}$. The amount of formazan dye generated by cellular dehydrogenase activity was measured by absorbance at $450 \mathrm{~nm}$ with a microplate reader.

\section{TUNEL assay}

For the TUNEL assay, the cells were harvested and washed with PBS, fixed and permeabilized, and then TUNEL-labeled using a One Step TUNEL Apoptosis Assay Kit (Beyotime, China) as instructed. The total percentage of apoptotic cells was estimated by determining 
the percentage of cells with positive TUNEL staining in five randomly selected fields from each slide under a microscope.

\section{TH staining}

After treatment, primary DA cultures were washed with PBS and fixed with $4 \%$ paraformaldehyde (PFA) for $15 \mathrm{~min}$. Then, the cells were blocked in PBS with $5 \%$ horse serum and $0.03 \%$ Triton X-100 for $1 \mathrm{~h}$. Immunocytochemistry was performed using mouse anti-tyrosine hydroxylase monoclonal antibody (Sigma). The percentage of cells with positive $\mathrm{TH}$ staining was estimated in five randomly selected fields under a fluorescent microscope.

Experimental animals were anesthetized and perfused transcardially with $0.9 \%$ saline followed by $4 \%$ PFA. Brain samples were postfixed with $4 \%$ PFA overnight and equilibrated in $15 \%$ and $30 \%$ sucrose. Coronal sections of $40 \mu \mathrm{m}$ were prepared with a sliding microtome. Immunohistochemistry was performed using mouse antityrosine hydroxylase monoclonal antibody. Unbiased quantification of $\mathrm{TH}$-immunoreactive neurons in the $\mathrm{SN}$ was performed according to the optical fractionator principle (Gundersen et al., 1988).

\section{Intracerebral injection}

Animals received two $2.5-\mu \mathrm{L}$ stereotaxic injections of $2.0 \mu \mathrm{g} / \mu \mathrm{L}$ $6-\mathrm{OHDA}$ (total dose $=10 \mu \mathrm{g} 6-\mathrm{OHDA}$ ) delivered at a rate of $0.5 \mu \mathrm{L} / \mathrm{min}$ and the syringe was left in place for an extra $5 \mathrm{~min}$ and then withdrawn gently, and the skin was sutured. Striatum injection coordinates were as follows: site 1: $\mathrm{AP}+1.3, \mathrm{ML}-2.8, \mathrm{DV}-4.5$; site 2: $\mathrm{AP}-0.6, \mathrm{ML}-4.0$, $\mathrm{DV}-5.5$; and tooth bar set at -3.2 . The lesion was allowed to progress for 1-3 weeks after that animals were killed for further analyses.

The vesicles $(1 \mu \mathrm{g} / \mu \mathrm{L}$, protein concentration) were injected at two sites (site 1: AP -4.8, ML -2.0, DV -7.1; site 2: AP -5.5, ML -1.9, DV -7.0) stereotaxically over the SN $(2.5 \mu \mathrm{L} /$ site $)$. Injection rate was $0.5 \mu \mathrm{L} / \mathrm{min}$ and the needle was left in place for $10 \mathrm{~min}$ before it was slowly retracted from the brain.

\section{Behavioral analysis}

The animals were tested for apomorphine-induced $(0.2 \mathrm{mg} / \mathrm{kg}$, i.p.) turning 1, 2 and 3 weeks after intracerebral injection. Only the rats that exhibited a mean rotation toward the healthy side at least 6.0 full body turns per min were considered as disease onset.

\section{Statistical analysis}

All experiments were performed in triplicate. Data were presented as the mean \pm SEM; statistical significance of difference ( $P$ value) was assessed using Student's $t$-test by GraphPad Prism 5 software (GraphPad, San Diego, CA, USA), and $P<0.05$ was considered significant.

\section{ACKNOWLEDGEMENTS}

This work was supported by grants from the National Natural Science Foundation of China (Grant Nos. 31000478, 81170309 and 31471019) and the National Basic Research Program (973 Program) (No. 2011CB504803).

\section{ABBREVIATIONS}

6-OHDA, 6-Hydroxydopamine hydrobromide; DA, dopamine; DMEM/F-12, Dulbecco's Modified Eagle Medium/Nutrient Mixture F-12; EM, electron microscope; FACS, Fluorescence Activated Cell Sorter; FBS, Fetal Bovine Serum; HBSS, Hank's Balanced Salt Solution; LPS, Lipopolysaccharide; MiRNAs, microRNAs; MPP+, 1-Methyl-4-phenylpyridinium iodide; MVs, microvesicles; $P D$, Parkinson's disease; PFA, paraformaldehyde; qPCR: quantitative real-time PCR; SN, substantia nigra; SVs, shedding vesicles; $\mathrm{TH}$, tyrosine hydroxylase; VM, ventral mesencephalon.

\section{COMPLIANCE WITH ETHICS GUIDELINES}

Susu Mao, Qi Sun, Hui Xiao, Chenyu Zhang and Liang Li declare that they have no conflict of interest. All institutional and national guidelines for the care and use of laboratory animals were followed.

\section{OPEN ACCESS}

This article is distributed under the terms of the Creative Commons Attribution 4.0 International License (http://creativecommons.org/ licenses/by/4.0/), which permits unrestricted use, distribution, and reproduction in any medium, provided you give appropriate credit to the original author(s) and the source, provide a link to the Creative Commons license, and indicate if changes were made.

\section{REFERENCES}

Al-Nedawi K, Meehan B, Rak J (2009) Microvesicles: messengers and mediators of tumor progression. Cell cycle 8:2014-2018

Antonucci F, Turola E, Riganti L, Caleo M, Gabrielli M, Perrotta C, Novellino L, Clementi E, Giussani P, Viani P et al (2012) Microvesicles released from microglia stimulate synaptic activity via enhanced sphingolipid metabolism. EMBO J 31:1231-1240

Barbagallo D, Piro S, Condorelli AG, Mascali LG, Urbano F, Parrinello N, Monello A, Statello L, Ragusa M, Rabuazzo AM et al (2013) miR-296-3p, miR-298-5p and their downstream networks are causally involved in the higher resistance of mammalian pancreatic alpha cells to cytokine-induced apoptosis as compared to beta cells. Bmc Genomics 14:62

Bianco F, Perrotta C, Novellino L, Francolini M, Riganti L, Menna E, Saglietti L, Schuchman EH, Furlan R, Clementi E et al (2009) Acid sphingomyelinase activity triggers microparticle release from glial cells. EMBO J 28:1043-1054

Chen Y, Vartiainen NE, Ying W, Chan PH, Koistinaho J, Swanson RA (2001) Astrocytes protect neurons from nitric oxide toxicity by a glutathione-dependent mechanism. J Neurochem 77:16011610

Forno LS, DeLanney LE, Irwin I, Di Monte D, Langston JW (1992) Astrocytes and Parkinson's disease. Prog Brain Res 94:429-436

Furman JL, Norris CM (2014) Calcineurin and glial signaling: neuroinflammation and beyond. J Neuroinflamm 11:158

Gundersen HJ, Bagger P, Bendtsen TF, Evans SM, Korbo L, Marcussen N, Moller A, Nielsen K, Nyengaard JR, Pakkenberg B et al (1988) The new stereological tools: disector, fractionator, 
nucleator and point sampled intercepts and their use in pathological research and diagnosis. APMIS 96:857-881

Gupta A, Pulliam L (2014) Exosomes as mediators of neuroinflammation. J Neuroinflamm 11:68

Hirsch EC, Hunot S, Damier P, Brugg B, Faucheux BA, Michel PP, Ruberg M, Muriel MP, Mouatt-Prigent A, Agid Y (1999) Glial cell participation in the degeneration of dopaminergic neurons in Parkinson's disease. Adv Neurol 80:9-18

Hockenbery DM, Oltvai ZN, Yin XM, Milliman CL, Korsmeyer SJ (1993) Bcl-2 functions in an antioxidant pathway to prevent apoptosis. Cell 75:241-251

Hu G, Yao H, Chaudhuri AD, Duan M, Yelamanchili SV, Wen H, Cheney PD, Fox HS, Buch S (2012) Exosome-mediated shuttling of microRNA-29 regulates HIV Tat and morphine-mediated neuronal dysfunction. Cell death Dis 3:e381

Janowska-Wieczorek A, Wysoczynski M, Kijowski J, Marquez-Curtis L, Machalinski B, Ratajczak J, Ratajczak MZ (2005) Microvesicles derived from activated platelets induce metastasis and angiogenesis in lung cancer. Int $\mathrm{J}$ Cancer 113:752-760

Jung HJ, Suh $Y$ (2014) Circulating miRNAs in ageing and ageingrelated diseases. J Genet Genomics 41:465-472

Keller S, Sanderson MP, Stoeck A, Altevogt P (2006) Exosomes: from biogenesis and secretion to biological function. Immunol Lett 107:102-108

Lee KW, Im JY, Woo JM, Grosso H, Kim YS, Cristovao AC, Sonsalla PK, Schuster DS, Jalbut MM, Fernandez JR et al (2013) Neuroprotective and anti-inflammatory properties of a coffee component in the MPTP model of Parkinson's disease. Neurotherapeutics 10:143-153

Lei SL, Zhao H, Yao HL, Chen Y, Lei ZD, Liu KJ, Yang Q (2014) Regulatory roles of microRNA-708 and microRNA-31 in proliferation, apoptosis and invasion of colorectal cancer cells. Oncol Lett 8:1768-1774

Li XZ, Bai LM, Yang YP, Luo WF, Hu WD, Chen JP, Mao CJ, Liu CF (2009) Effects of IL-6 secreted from astrocytes on the survival of dopaminergic neurons in lipopolysaccharide-induced inflammation. Neurosci Res 65:252-258

Liu C, Zhou CF, Gao F, Cai SY, Zhang C, Zhao LQ, Zhao F, Cao F, Lin J, Yang YY et al (2011) MiR-34a in Age and Tissue Related Radio-Sensitivity and Serum miR-34a as a Novel Indicator of Radiation Injury. Int J Biol Sci 7:221-233

Liu Y, Zhao L, Li D, Yin Y, Zhang CY, Li J, Zhang Y (2013) Microvesicle-delivery miR-150 promotes tumorigenesis by upregulating VEGF, and the neutralization of miR-150 attenuate tumor development. Protein Cell 4:932-941

Malavolta M, Basso A, Piacenza F, Costarelli L, Giacconi R, Mocchegiani E (2013) Peripheral mononuclear cell rejuvenation for senescence surveillance in Alzheimer disease. Curr Pharm Des 19:1720-1726

Morel O, Toti F, Hugel B, Freyssinet JM (2004) Cellular microparticles: a disseminated storage pool of bioactive vascular effectors. Curr Opin Hematol 11:156-164

Niranjan R, Nath C, Shukla R (2010) The mechanism of action of MPTP-induced neuroinflammation and its modulation by melatonin in rat astrocytoma cells, C6. Free Radical Res 44:13041316
Oeckl P, Lattke M, Wirth T, Baumann B, Ferger B (2012) Astrocytespecific IKK2 activation in mice is sufficient to induce neuroinflammation but does not increase susceptibility to MPTP. Neurobiol Dis 48:481-487

Saijo K, Winner B, Carson CT, Collier JG, Boyer L, Rosenfeld MG, Gage FH, Glass CK (2009) A Nurr1/CoREST pathway in microglia and astrocytes protects dopaminergic neurons from inflammation-induced death. Cell 137:47-59

Saura J, Pares M, Bove J, Pezzi S, Alberch J, Marin C, Tolosa E, Marti MJ (2003) Intranigral infusion of interleukin-1beta activates astrocytes and protects from subsequent 6-hydroxydopamine neurotoxicity. J Neurochem 85:651-661

Scherr M, Elder A, Battmer K, Barzan D, Bomken S, Ricke-Hoch M, Schroder A, Venturini L, Blair HJ, Vormoor J et al (2014) Differential expression of miR-17 92 identifies BCL2 as a therapeutic target in BCR-ABL-positive B-lineage acute lymphoblastic leukemia. Leukemia 28:554-565

Shantsila E, Kamphuisen PW, Lip GY (2010) Circulating microparticles in cardiovascular disease: implications for atherogenesis and atherothrombosis. J Thromb Haemost 8:2358-2368

Song T, Zhang X, Zhang L, Dong J, Cai W, Gao JP, Hong BF (2013) miR-708 promotes the development of bladder carcinoma via direct repression of Caspase-2. J Cancer Res Clin 139:1189-1198 Stromberg I, Bjorklund H, Dahl D, Jonsson G, Sundstrom E, Olson L (1986) Astrocyte responses to dopaminergic denervations by 6-hydroxydopamine and 1-methyl-4-phenyl-1,2,3,6-tetrahydropyridine as evidenced by glial fibrillary acidic protein immunohistochemistry. Brain Res Bull 17:225-236

Sun L, Wu Z, Shao Y, Pu Y, Miu W, Yao J, Wu Y, Yang Z (2012) MicroRNA-34a suppresses cell proliferation and induces apoptosis in U87 glioma stem cells. Technol Cancer Res Treat 11:483-490

Vlassov AV, Magdaleno S, Setterquist R, Conrad R (2012) Exosomes: current knowledge of their composition, biological functions, and diagnostic and therapeutic potentials. Biochim Biophysica Acta 1820:940-948

Wang X, Liu P, Zhu H, Xu Y, Ma C, Dai X, Huang L, Liu Y, Zhang L, Qin C (2009) miR-34a, a microRNA up-regulated in a double transgenic mouse model of Alzheimer's disease, inhibits bcl2 translation. Brain Res Bull 80:268-273

Yang J, Liu X, Bhalla K, Kim CN, Ibrado AM, Cai J, Peng TI, Jones DP, Wang X (1997) Prevention of apoptosis by Bcl-2: release of cytochrome c from mitochondria blocked. Science 275:11291132

Zhang Y, Liu D, Chen X, Li J, Li L, Bian Z, Sun F, Lu J, Yin Y, Cai X et al (2010) Secreted monocytic miR-150 enhances targeted endothelial cell migration. Molecular cell 39:133-144

Zhou Y, Xiong M, Niu J, Sun Q, Su W, Zen K, Dai C, Yang J (2014) Secreted fibroblast-derived miR-34a induces tubular cell apoptosis in fibrotic kidney. J Cell Science 127:4494-4506

Zhu H, Fan GC (2011) Extracellular/circulating microRNAs and their potential role in cardiovascular disease. Am J Cardiovasc Dis 1:138-149

Zomer A, Vendrig T, Hopmans ES, van Eijndhoven M, Middeldorp JM, Pegtel DM (2010) Exosomes: Fit to deliver small RNA. Communicative Integr Biol 3:447-450 